\title{
EDITORIAL
}

\section{MRSA as a cause of community-acquired pneumonia}

\author{
A. Nakou*, M. Woodhead ${ }^{\star, *}$ and A. Torres
}

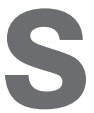
taphylococcus aureus is a species of bacterium that can cause a broad variety of infections, ranging from minor skin infections to severe pneumonia and sepsis. The genetic adaptation of $S$. aureus has led to a multidrug-resistant pathogen, meticillin-resistant $S$. aureus (MRSA) after the introduction of meticillin (previously methicillin) into clinical practice in the 1960s [1]. MRSA is resistant to $\beta$-lactam antibiotics, including penicillin and cephalosporins. Resistance is mediated by penicillin binding protein $2 \mathrm{a}$, a penicillin binding protein encoded by the mecA gene that permits growth in the presence of meticillin. The mecA gene is situated in the staphylococcal cassette chromosome SCCmec. Initially, MRSA was only a nosocomial pathogen, i.e. healthcare-associated MRSA. It is associated with severe invasive disease [2] and tends to have multidrug resistance. It carries SCC chromosome type II (SCCmec type II) [3].

In the mid 1990s, MRSA began to be detected in the community, i.e. community-acquired MRSA (CA-MRSA). It is the leading cause of identifiable skin and soft tissue infections seen in USA emergency rooms [4]. CA-MRSA (and meticillinsusceptible $S$. aureus (MSSA)) may be associated with the presence of Panton Valentine leukocidin (PVL), a twocomponent staphylococcal membrane toxin that targets leucocytes [5] and frequently carries SCC mec type V or IV. PVL isolates have been linked to severe infections and necrotising pneumonia [6].

Published data has suggested that MRSA was not a frequent cause of community-acquired pneumonia (CAP), although with increasing incidence over the past few years the situation is now less clear. Now, a systematic study of both case series and case reports of CA-MRSA pneumonia, published in the present issue of the European Respiratory Journal, has estimated the incidence at $0.51-0.64$ cases per 100,000 population [7]. Since the CAP incidence is between 100 and 1,000 per 100,000 [8], the frequency of CA-MRSA CAP can be estimated to be between one in 200 and one in 2,000 cases of CAP.

MRSA CAP appears to be a severe disease, with multilobar shadowing in three quarters of patients, $75-85 \%$ reaching the intensive care unit (ICU) and death occurring in between $20 \%$ and $60 \%$ in the article by VARDAKAS et al. [7]. However, the resistance profile of the pathogen does not necessarily determine the severity of disease caused by that organism.

\footnotetext{
*Dept of Respiratory Medicine, Manchester Royal Infirmary, and \#University of Manchester Manchester, UK. "Clinic de Pneumologia I Cirurgia Toràcica, Hospital Clinic I Provincial de Barcelona, Barcelona, Spain.

CORRESPONDENCE: M. Woodhead, Dept of Respiratory Medicine, Manchester Royal Infirmary, Oxford Road, Manchester M13 9WL, UK. E-mail: mark.woodhead@cmft.nhs.uk
}

Although the majority of CA-MRSA infections involving skin and soft tissue most likely occur independent of PVL toxin production [9], PVL is required for lung tissue necrosis and inflammation, as present in the rare, but frequently fatal, cases of necrotising pneumonia [10]. Change to the initial empirical antibiotic regimen was deemed to be appropriate in most cases from the published articles.

The frequency in the more severely ill admitted to the hospital and ICU is much more difficult to assess. The frequency of S. aureus CAP in the ICU averages $\sim 10 \%$, and $\sim 10 \%$ of adults admitted with CAP are managed in the ICU, but rates of both vary enormously from country to country.

Compared with nosocomial MRSA, CA-MRSA isolates are commonly sensitive to antibiotics such as clindamycin, fluoroquinolones, trimethoprim-sulfamethoxazole, tetracyclines and rifampicin, although some resistant isolates are emerging [11]. There is evidence of increasing prevalence of asymptomatic colonisation among children and adults in the community $[12,13]$. The strain most often isolated in the community outbreaks was USA300 [14], which is typically resistant to $\beta$-lactam and macrolide antimicrobial agents and contains genes for PVL toxin [15]. USA300 isolate has also been described among "men who have sex with men" [16]. Although CA-MRSA is sensitive to several antibiotics (clindamycin, trimethoprim-sulphamethoxazole and tetracyclines) the optimal treatment is not yet established. The 2007 Infectious Diseases Society of America/American Thoracic Society guidelines recommend vancomycin or linezolid [17]. Linezolid, an agent from the oxazolidone family, is active against all CA-MRSA isolates but prolonged administration increases the likelihood of resistance through the accumulation of mutations in multiple copies of the 23s ribosomal RNA $S$. aureus gene [18]. Vancomycin is still considered first-line treatment for patients with invasive infection. Strains of MRSA with high levels of resistance to vancomycin are evolving [4]. Observational reports have used these antibiotics (vancomycin or linezolid) in combination with clindamycin. Owing to the increasing importance of CA-MRSA, animal studies and human randomised trials are needed to find the best antibiotic treatment. The currently recommended empirical therapy for CAP, which consists of a $\beta$-lactam with a macrolide or fluoroquinolone for severe $\mathrm{CAP}$, may not provide adequate protection against MRSA. The frequency of CA-MRSA CAP overall should have no influence on guideline antibiotic recommendations; however, if the above projections are correct the frequency in those reaching the ICU could be enough to impact on guideline recommendations. The many excellent studies of the microbial aetiology of CAP in hospitals 
and the ICU must be supplemented by new studies to bridge this knowledge gap.

Post-influenza staphylococcal pneumonia has been reported during influenza pandemics and epidemics for the past century. There is evidence of mechanisms indicating influenza interaction with $S$. aureus: an influenza-induced increase in S. aureus-specific adhesion throughout the respiratory tract and S. aureus-specific proteases, which may increase influenza viral replication [19, 20]. During the 2003-2004 influenza season, 15 cases of pneumonia caused by S. aureus. MSSA and MRSA were reported to the Centers for Disease Control and Prevention (CDC) [21]. In 2007, the CDC received reports of 10 cases of MRSA CAP, including six deaths among previously healthy individuals [22]. In the present series of MRSA CAP, $38 \%$ had documented evidence of influenza infection and $57 \%$ had symptoms consistent with influenza [7]. MRSA should remain in the differential diagnosis of severe CAP occurring during the influenza season, especially in those with cavitary infiltrates and in those with a history of MRSA infection. This could be of great importance in the ongoing pandemic of swine-related H1N1 influenza virus infection.

As the incidence of CA-MRSA rises, either as a cause of skin and soft tissue infection or as a cause of CAP, physicians should take into consideration traditional risk factors for MRSA infections, especially if resistant clones are involved and in severe CAP cases: recent hospitalisation, surgery, dialysis, residence in a long-term care facility in the 12 months preceding the culture, invasive device, and history of MRSA infection or colonisation. In addition, evidence of skin and soft tissue infection should now be sought. Rapid tests for confirmation of $S$. aureus infection need to be supplemented by rapid tests for toxin-producing strains.

The rarity of such infections and the practical difficulties of such trials mean that comparative randomised antibiotic trials in CA MRSA CAP will probably never be carried out. Additional data from animal studies and trials in skin and soft tissue infection are required to guide antibiotic recommendations and therapy for this difficult infection. A review series on MRSA and the lung is launched in this issue of the European Respiratory Journal [23] and will develop these topics further as the series continues.

\section{STATEMENT OF INTEREST}

Statements of interest for M. Woodhead and A. Torres can be found at www.erj.ersjournals.com/misc/statements.dtl

\section{REFERENCES}

1 Daum RS. Skin and soft tissue infections caused by methicillinresistant Staphylococcus aureus. N Engl J Med 2007; 357: 380-390.

2 Wisplinoff $\mathrm{H}$, Biscoff $\mathrm{T}$, Tallent SM, et al. Nosocomial bloodstream infections in US hospitals: analysis of 24,179 cases from a prospective nationwide surveillance study. Clin Infect Dis 2004; 39: 309-317.

3 Naimi TS, LeDell KH, Como-Sabetti K, et al. Comparison of community and health care-associated methicillin-resistant Staphylococcus aureus infection. JAMA 2003; 290: 2976-2984.
4 Moran GJ, Krishnaden A, Gorwitz RJ, et al. Methicillin-resistant $S$. aureus infections among patients in the emergency department. N Engl J Med 2006; 355: 666-674.

5 Lina G, Piemont Y, Godail-Gamot F, et al. Involvement of Panton Valentine leucocidin-producing Staphylococcus aureus in primary skin infections and pneumonia. Clin Infect Dis 1999; 29: 1128-1132.

6 Gillet Y, Issartel B, Vanhems P, et al. Association between Staphylococcus aureus strains carrying gene for Panton Valentine leucocidin and highly lethal necrotizing pneumonia in young immunocompent patients. Lancet 2002; 359: 753-759.

7 Vardakas KZ, Matthaiou DK, Falagas ME. Incidence, characteristics and outcomes of patients with severe community acquiredMRSA pneumonia. Eur Respir J 2009; 34: 1148-1158.

8 Woodhead M. The European vision of community-acquired pneumonia. Semin Respir Crit Care Med 2009; 30: 136-145.

9 Wang R, Braughton KR, Kretschmer D, et al. Identification of novel cytolytic peptides as key virulence determinants for communityassociated MRSA. Nat Med 2007; 13: 1510-1514.

10 Labandeira-Rey M, Couzon F, Boisset S, et al. Staphylococcus aureus Panton-Valentine leukocidin causes necrotizing pneumonia. Science 2007; 315: 1130-1133.

11 Arias CA, Murray BE. Antibiotic resistant bugs in the 21st century - a clinical super-challenge. N Engl J Med 2009; 360: 439-443.

12 Creech CB II, Kernodle DS, Alsentzer A, et al. Increasing rates of nasal carriage of methicillin-resistant Staphylococcus aureus in healthy children. Pediatr Infect Dis J 2005; 24: 617-621.

13 Hidron AI, Kourbatova EV, Halvosa JS, et al. Risk factors for colonization with methicillin-resistant Staphylococcus aureus (MRSA) in patients admitted to an urban hospital: emergence of community-associated MRSA nasal carriage. Clin Infect Dis 2005; 41: 159-166.

14 Klevens RM, Morrison MA. Invasive methicillin resistant Staphylococcus aureus infections in the United States. JAMA 2007; 298: 1763-1771.

15 Tenover FC, McDougal LK, Goering RV, et al. Characterization of a strain of community-associated methicillin-rezistant Staphylococcus aureus widely disseminated in the United States. J Clin Microbiol 2006; 44: 108-118.

16 Diep BA, Champers HF, Graber CJ, et al. Emergence of multidrug resistant community associated, methicillin-resistant Staphylococcus aureus clone USA300 in men who have sex with men. Ann Intern Med 2008; 148: 249.

17 Mandell LA, Wunderink RG, Anzueto A, et al. Infectious Diseases Society of America/American Thoracic Society consensus guidelines on the management of community-acquired pneumonia in adults. Clin Infect Dis 2007; 44: Suppl. 2, S27-S72.

18 Peeters MJ, Sarrria JC. Clinical characteristics of linezolid-resistant Staphylococcus aureus infections. Am J Med Sci 2005; 330: 102-104.

19 Sanford BA, Ramsay MA. Bacterial adherence to the upper respiratory tract of ferrets infected with influenza A virus. Proc Soc Exp Biol Med 1987; 185: 120-128.

20 Tashiro M, Ciborowski P, Reinacher M, et al. Synergistic role of staphylococcal proteases in the induction of influenza virus pathogenicity. Virology 1987; 157: 421-430.

21 Hageman JC, Uyeki TM, Francis JS, et al. Severe community acquired pneumonia due to Staphylococcus aureus, 2003-04 influenza season. Emerg Infect Dis 2006; 12: 894-899.

22 Centers for Disease Control and Prevention. Severe methicillinresistant Staphylococcus aureus community-acquired pneumonia associated with influenza - Louisiana and Georgia, December 2006-January 2007. MMWR Morb Mortal Wkly Rep 2007; 56: 325-329.

23 Pantosti A, Venditti M. What is MRSA? Eur Respir J 2009; 34: 1190-1196. 